\title{
REVIEW ON Classification BASEd ON ARTIFICIAL NEURAL NETWORKS
}

\author{
Saravanan $\mathrm{K}^{1}$ and S. Sasithra ${ }^{2}$ \\ ${ }^{1}$ Assistant Professor/Department of Computer Engineering, Erode Sengunthar \\ Engineering College, Erode, India \\ ${ }^{2}$ Department of Computer Engineering, Erode Sengunthar Engineering College, Erode, \\ India
}

\begin{abstract}
Artificial neural networks (ANN) consider classification as one of the most dynamic research and application areas. ANN is the branch of Artificial Intelligence (AI). The neural network was trained by back propagation algorithm. The different combinations of functions and its effect while using ANN as a classifier is studied and the correctness of these functions are analyzed for various kinds of datasets. The back propagation neural network (BPNN) can be used as a highly successful tool for dataset classification with suitable combination of training, learning and transfer functions. When the maximum likelihood method was compared with backpropagation neural network method, the BPNN was more accurate than maximum likelihood method. A high predictive ability with stable and well functioning BPNN is possible. Multilayer feed-forward neural network algorithm is also used for classification. However BPNN proves to be more effective than other classification algorithms.
\end{abstract}

\section{KEYWORDS}

Back propagation algorithm, Maximum Likelihood method, Multilayer feed-forward neural network.

\section{INTRODUCTION}

A neural network model which is the branch of artificial intelligence is generally referred to as artificial neural networks (ANNs). ANN teaches the system to execute task, instead of programming computational system to do definite tasks. To perform such tasks, Artificial Intelligence System (AI) is generated. It is a pragmatic model which can quickly and precisely find the patterns buried in data that replicate useful knowledge. One case of these AI models is neural networks. AI systems should discover from data on a constant basis. In the areas of medical diagnosis relationships with dissimilar data, the most available techniques are the Artificial Intelligence techniques.

An artificial neural network is made up of many artificial neurons which are correlated together in accordance with explicit network architecture. The objective of the neural network is to convert the inputs into significant outputs. The teaching mode can be supervised or unsupervised. Neural Networks learn in the presence of noise.

ANNs found their usage in many areas suc as,

- Bankruptcy prediction

DOI:10.5121/ijasa.2014.2402 
- Speech recognition

- Product inspection

- Fault detection



Fig1. Artificial Neural Network

\section{EXISTING SYSTEM}

\subsection{Back propagation Neural Network}

Artificial neural networks (ANN) consider classification as one of the most dynamic research and application areas. The major disadvantage in using ANN is to find the most appropriate grouping of training, learning and transfer function for classifying the data sets with growing number of features and classified sets. The different combinations of functions and its effect while using ANN as a classifier is studied and the correctness of these functions are analyzed for various kinds of datasets.

The real world problems which are represented by multidimensional datasets are taken from medical background. The classification and clustering of these data sets are significant. The data set is divided into training set and testing set and it has no usage in the training process. The results are produced with the help of these datasets and it is used for testing. The training set is taken from $2 / 3^{\text {rd }}$ of the dataset and the remaining has been taken as test set. This is made through the assessment of the accuracy achieved through testing against these data sets. Then the network is simulated with the same data.

The back propagation algorithm trains the neural network. Gradient descent method (GDM) was used to decrease the mean squared error between network output and the actual error rate. The following parameters are considered to measure the efficiency of the network,

- Rate of convergence

- No of epochs taken to converge the network.

- The calculated Mean Square Error (MSE).

With the appropriate combination of training, learning and transfer functions the dataset classification uses the most successful tool called back propagation neural network. The combination of TRAINLM, LEARNGDM and LOGSIG mentioned in the proposed work works 
better for relatively smaller datasets but the combination TRAINSCG LEARNGDM and LOGSIG is successful for larger datasets.

\section{E. Hosseini Aria, J. Amini, M.R.Saradjian(2009) proposed a method for Classifying IRS-1D Satellite Images[4].}

The fitness of Back Propagation Neural Network (BPNN) for classification of remote sensing images based on three steps is proposed. As an initial step, from the measures of first order histogram measures the features are extracted.In the second step, feature classification based on BPNN is done, and in the third step the outcomes are compared with the maximum likelihood classification (MLC) method. The statistical features in this paper depend on the first-order distribution measure such as mean, standard-deviation, skewness, kurtosis, energy, and entropy. The network contains 3 layers. The input layer is fed with extracted features which contains 18 neurons.in the classification of IRS-1D satellite images six classes were used and the back ropagation neural network was trained on these classes. The whole image was classified using this trained network. The regions of Iran are taken for testing.

The IRS-1D satellite images uses Artificial neural network for the classification of images. The major problem with the classification of IRS data is to choose a better method for training. TrainLM method has been implemented on using back propagation neural networks algorithm on IRS images.

There are various training algorithms for feed forward networks The gradient of the performance function is used by all the algorithms to find out how to fiddle with the weights to decrease the performance. The back propagation technique determines the gradient. This gradient performs computational backwards through the network. When the maximum likelihood method was compared with backpropagation neural network method, the BPNN was more accurate than maximum likelihood method.The overall preciseness in MLC method is $75.00 \%$ whereas in BPNN method is $85.19 \%$.

\section{Helena Grip, Fredrik(2003) proposed Whiplash-Associated Disorders by classifying of neck movement patterns[5].}

A novel method for the classification of neck movement patterns related to Whiplash-associated disorders (WAD) using a flexible back propagation neural network (BPNN) is studied. WAD is a common diagnosis after neck trauma, mainly caused by rear-ends car accidents. Since physical injuries cannot be detected with the current imaging techniques, the diagnosis can be complex to make. The dynamic range of the neck is often visually detected in patients with neck pain, but this is a biased measure, and a more intentional decision support system, that gives a consistent and more complete analysis of neck movement pattern, is needed. The estimation of the prognostic ability of a BPNN, using neck movement variables as input is the main objective of the paper. The collection of three-dimensional (3-D) neck movement data from 59 subjects with WAD and 56 control subjects is made with a proReflex system. Rotation angle and angular velocity were measured using the direct helical axis method and motion variables and the results are extracted. To increase the performance of BPNN a principal component analysis was performed which reduces data. BPNNs with six hidden nodes had a yield of 0.89 , a sensitivity of 0.90 and a specificity of 0.88 , which are very hopeful results. The results were predicted from the neck movement analysis. The result was combined with a neural network where the origin of decision support system is constructed, which classifies the suspected WAD. 
International Journal of Ambient Systems and Applications (IJASA) Vol.2, No.4, December 2014

The flexible back propagation neural network (BPNN) resulted in a correct calculation for 84 percent of the control subjects and 89 percent of the WAD, showing that a BPNN could be appropriate for predicting motion characteristics. The presented method is very hopeful as an aid to determine whether a patient with suspected WAD has a neck movement pattern that deviates from that found in control subjects. A few perceptive variables seems to amplify the efficiency on abbreviating the results. A high predictive ability with stable and well functioning BPNN is presented using early stopping method.

Yu-guo Wang, Hua-peng $\operatorname{Li}(2010)$ proposed classification of Remote sensing images using artificial neural network[6].

Artificial neural network (ANN) is a significant part of artificial intelligence, It has been extensively used in the research field of remote sensing classification. The wetlands remote sensing classification based on ANN is complicated, because of the intricate feature of wetlands areas. The remote sensing image supervised classification is carried out on the training samples. The clarity is examined and it was found that it is hard to guarantee because it will have an effect on the classification results. This article proposed a method for sample purification to filter the training samples based on statistical analysis theory for enhanced wetlands remote sensing classification based on ANN. The BP ANN with a nonlinear mapping function gives better classification results for intricate areas. The TM image of Honghe Wetlands National Nature Reserve is chosen for classification. First, the statistical analysis theory to eradicate noise in training samples is used. Then the original samples and purified samples are used to train the BP ANN individually and created two classification maps of TM image based on two trained BP ANN. At last, the classification accuracy between the two maps is compared. The statistical analysis method for purifying training samples for remote sensing classification based on BP ANN is performed. The experiments showed that it was an efficient method to develop image classification.

Guoqiang Peter Zhang(2000) proposed the use of harmony search and back propagation based ANN to classify breast cancer data.

Breast cancer is one of the most commonly seen cancer types. Analysis of breast cells characteristics has great importance in diagnosis, treatment and following of this disease. Through two different types of ANN algorithm 699 instances of breast cancer data which is present in UCI are classified. In this study, abundant algorithms are used for training of artificial neural network. In this study, two algorithms namely harmony search and back propagation algorithms are used to train feed forward artificial neural network. While classifying the performance values of classification are found by means of Accuracy/SSE/Regression parameters. The performance values of back propagation are achieved as 94.1/0.007/0.92 whereas the results obtained by the harmony search algorithm are 97.57/0.005/0.96 respectively. With the help of harmony search based ANN algorithm breast cancer data are classified for the first time with this study.

\subsection{MULTILAYER FEEDFORWARD NETWORK}

\section{Guoqiang Peter Zhang(2000) proposed Neural Networks for Classification[7].}

Classification is one of the most dynamic exploratory and application areas of neural network. The issues of posterior probability estimation, link between neural and conventional classifiers, learning and generalization tradeoff in classification, feature variable selection, effect of misclassification costs are studied. 
Multilayer feed-forward network is used for classification. Although many procedures are available in traditional statistical classification, the usefulness depends on assumptions and circumstances. Inorder to overcome the above disadvantage, neural networks are used since they are,

- self-adaptive methods,

- universal functional approximators and

- non-linear models.

Many problems still stay unsettled even though neural networks have grown rapidly. As represented prior, many researches should be dedicated to develop more successful and proficient methods in neural model identification, feature variable selection, classifier combination, and uneven misclassification treatment. As a practical decision making tool, systematic evaluation and comparison of neural networks with other traditional classifiers need to be done. The lacks of comparisons between other classifying methods and neural network have been proposed by some authors in the existing literature. Due to the lack of comparisons, mixed results are produced and reported in pragmatic studies.

There are other topics which are related to neural classification. The topics include sample size issues, Bayesian analysis, wavelet network and network training model design and selection. Some applications of neural network have been reviewed formerly and the problems mentioned above are general to all applications. Abundant research opportunities are available in neural classifiers. More research actions are generated by neural network classification because of its multidisciplinary nature and brings about more productive outcomes in the future.

\section{Abid Ali, Olaf Magnor and Matthias Schultalbers proposed Neural Network Based Pattern Recognition Technique which detects the misfire in gasoline engines[9]}

The application of ANN to misfire detection in gasoline engines is studied. A feed-forward multiple layer neural network is used for the classification of firing and misfiring events. Importance is given on the transaction between performance, computational cost and implement ability and the system is applied on a production electronic control unit (ECU). The technique is used to identify misfire events over the whole range of operation defined by official on-board diagnosis (OBD) regulations and it is applied to a six-cylinder gasoline engine. Experimental results on a passenger car are presented.

Misfire occurs because of some error in the combustion process. This error occurs because of some insufficiency in ignition, injection or compression system of the engine or due to a substandard fuel. In addition to their disagreeable effects on the drive quality, misfires in gasoline engines amplify pollutants in exhaust gases and causes harm to the components. Misfire due to some defect in ignition system will enlarge unburned mixture of hydrocarbons and oxygen in the raw exhausts causing an increased exothermic reaction in the catalyst and increased $\mathrm{HC}$ and $\mathrm{CO}$ emissions.

The investigation of realistic application of a neural network based pattern recognition technique to misfire detection in gasoline engines is done. The difficulty of misfire detection is framed as a pattern recognition problem. The classification of firing and misfiring events is done with the help of feed-forward multiple-layer neural network. To detect the misfire events in six-cylinder gasoline engines, the developed technique is applied over a wide range of operation. The results 
International Journal of Ambient Systems and Applications (IJASA) Vol.2, No.4, December 2014

are produced in this section. The results show that the developed technique detects all patters of misfire.

\section{R. Furferi, L. Governi and Y. Volpe(2011) proposed the classification of car seat fabrics[10].}

Car seat fabrics are exclusively shaped textiles. A first-rate car seat fabric needs a little quantity of spots and discolored areas. There are large and low number of spots in the fabrics which characterizes them. The above mentioned fabric types are considered to be of poor quality. Most of them is branded by a sponged-like appearance, characterized by spots and slightly discolored areas. The human experts with the help of manual inspection and classification perform spongedlike fabric grading. Though this manual classification and inspection proves to be valuable in fabric grading, the procedure is skewed and its results may differ depending on the operator skills.

The computer based tool which classifies sponged-like fabrics similar to the classification performed by skilled operators is shown here. Such a tool which is developed by an oppositely devised machine vision system, is able to extract a lot of numerical parameters characterizing the fabric veins and discolored areas. Since the Artificial Neural Network has to be trained, the parameters are used which classifies the fabrics based on quality. At last, a relationship between the ANN-based classification and the classification provided by fabric inspectors is done. The validation set composed by 65 sponged-like fabrics is tested and it shows that it is able to classify the fabrics into the correct quality class in $93.8 \%$ of the cases, similar to the selection provided by human operators.

With the aim of training a ANN able to correctly classify the fabrics, a series of parameters describing their appearance have been extracted by means of image processing-based algorithms; such data are used as a training, validation and testing set for the ANN. In detail, as described below, three kinds of parameters are extracted: colorimetric data, entropy curve and area subtended to the entropy curve.

Most of the methods evaluates the classification performance by defining a dimensionless parameter. A comparison between those methods and the proposed method was provided. For instance, the correlation between measured and forecasted classifications of colored textiles is stated to be in the range $0.85-0.98$. The classification error of textured objects is less than $10 \%$. These results are comparable with the ones provided by the present work, thus allowing to state that a satisfactory performance has been obtained.

\section{Problems in the Existing System}

The major disadvantage in using ANN is to find the most appropriate grouping of training, learning and transfer function for classifying the data sets with growing number of features and classified sets. The classification of IRS-1D satellite images is done using Artificial Neural Networks. The algorithm is used to categorize the imagery. The major problem with the classification of IRS data is to choose a better method for training. Abundant algorithms are used for training of artificial neural network. . Although many procedures are available in traditional statistical classification, the usefulness depends on assumptions and circumstances. In order to overcome the above disadvantage, neural networks are used. 
International Journal of Ambient Systems and Applications (IJASA) Vol.2, No.4, December 2014

Table1 Comparison of various classification methods based on artificial neural network

\begin{tabular}{|c|c|c|c|c|c|}
\hline SNO & Mechanism & Method used & Author & Advantages & Disadvantages \\
\hline 1 & $\begin{array}{l}\text { Neural network } \\
\text { for classification }\end{array}$ & $\begin{array}{l}\text { Probability } \\
\text { estimation for } \\
\text { Posterior }\end{array}$ & $\begin{array}{l}\text { Peter Zhang } \\
\text { Guoqiang }\end{array}$ & $\begin{array}{l}\text { The important } \\
\text { aspects is solving } \\
\text { classification } \\
\text { problems are } \\
\text { discussed }\end{array}$ & $\begin{array}{l}\text { lack of the } \\
\text { rigorous }\end{array}$ \\
\hline 2 & $\begin{array}{l}\text { ANN for dataset } \\
\text { classification }\end{array}$ & $\begin{array}{l}\text { Backpropagation } \\
\text { algorithm }\end{array}$ & $\begin{array}{l}\text { Rojalina } \\
\text { Priyadarshini }\end{array}$ & $\begin{array}{l}\text { The best } \\
\text { combination of } \\
\text { training, learning } \\
\text { and transfer } \\
\text { function for } \\
\text { classification of } \\
\text { datasets is found }\end{array}$ & $\begin{array}{l}\text { The combination } \\
\text { doesn't worked } \\
\text { for larger } \\
\text { datasets }\end{array}$ \\
\hline 3 & $\begin{array}{l}\text { Classification of } \\
\text { car seat fabrics }\end{array}$ & $\begin{array}{l}\text { Computer based } \\
\text { tool is used }\end{array}$ & $\begin{array}{l}\text { R.Furferi, L. } \\
\text { Governi }\end{array}$ & $\begin{array}{l}\text { Classified the } \\
\text { fabrics in } 93.8 \% \\
\text { of the cases }\end{array}$ & $\begin{array}{l}\text { Result varies } \\
\text { from operator to } \\
\text { operator }\end{array}$ \\
\hline 4 & $\begin{array}{l}\text { Classification of } \\
\text { IRS-1D satellite } \\
\text { images }\end{array}$ & $\begin{array}{l}\text { Backpropagation } \\
\text { algorithm }\end{array}$ & $\begin{array}{l}\text { E. Hosseini } \\
\text { Aria, J. Amini, } \\
\text { M.R.Saradjian }\end{array}$ & $\begin{array}{l}\text { BPNN is more } \\
\text { accurate }\end{array}$ & $\begin{array}{l}\text { Maximum } \\
\text { likelihood } \\
\text { classification is } \\
\text { less accurate } \\
\text { than BPNN }\end{array}$ \\
\hline 5 & $\begin{array}{l}\text { Classification of } \\
\text { neck movement } \\
\text { patterns }\end{array}$ & $\begin{array}{l}\text { Backpropagation } \\
\text { algorithm }\end{array}$ & $\begin{array}{l}\text { Helena Grip, } \\
\text { Fredrik Öhberg }\end{array}$ & $\begin{array}{l}\text { the predictive } \\
\text { ability of a BPNN, } \\
\text { using neck } \\
\text { movement } \\
\text { variables as input } \\
\text { is analysed and it } \\
\text { produced the } \\
\text { result with 90\% } \\
\text { accuracy }\end{array}$ & $\begin{array}{l}\text { data } \\
\text { concerning age, } \\
\text { proprioception, } \\
\text { muscle activity } \\
\text { are not discussed }\end{array}$ \\
\hline 6 & $\begin{array}{l}\text { Misfire detection } \\
\text { using NN }\end{array}$ & $\begin{array}{l}\text { Feed-forward } \\
\text { multiple layer NN }\end{array}$ & $\begin{array}{l}\text { Abid Ali, Olaf } \\
\text { Magnor }\end{array}$ & $\begin{array}{l}\text { It detects all } \\
\text { patterns of misfire }\end{array}$ & $\begin{array}{l}\text { potential } \\
\text { implementation } \\
\text { factors, like } \\
\text { fixed-point } \\
\text { operations, } \\
\text { quantization are } \\
\text { not discussed }\end{array}$ \\
\hline 7 & $\begin{array}{l}\text { Remote sensing } \\
\text { image } \\
\text { classification }\end{array}$ & $\begin{array}{l}\text { Backpropagation } \\
\text { algorithm }\end{array}$ & $\begin{array}{l}\text { Yu-guo Wang, } \\
\text { Hua-peng Li }\end{array}$ & $\begin{array}{l}\text { BP ANN with a } \\
\text { mapping function } \\
\text { is used and the } \\
\text { image } \\
\text { classification is } \\
\text { improved }\end{array}$ & $\begin{array}{l}\text { Not suitable for } \\
\text { wide spectral } \\
\text { distribution }\end{array}$ \\
\hline 8 & $\begin{array}{l}\text { Gene Expression } \\
\text { Data } \\
\text { Classification }\end{array}$ & Samples filtering & $\begin{array}{l}\text { Wutao } \\
\text { Chen,Huijuan } \\
\text { Lu,Mingyi } \\
\text { Wang }\end{array}$ & $\begin{array}{l}\text { The result is more } \\
\text { accurate and stable }\end{array}$ & $\begin{array}{l}\text { It is unstable for } \\
\text { single neural } \\
\text { network }\end{array}$ \\
\hline
\end{tabular}


International Journal of Ambient Systems and Applications (IJASA) Vol.2, No.4, December 2014

\section{CONCUlsion}

Artificial neural networks (ANN) say that the most active research and application an area is classification.The neural network was trained by back propagation algorithm. The dataset classification uses the most effective tool called back propagation neural network. The usage of Back Propagation Neural Network (BPNN) for classifying the images in remote sensing technology is also examined. When the maximum likelihood method was compared with backpropagation neural network method, the BPNN was more accurate than maximum likelihood method. The classification of neck movement patterns related to Whiplash-associated disorders (WAD) uses a resilient back propagation neural network (BPNN). The resilient back propagation neural network (BPNN) resulted in a correct prediction for 84 percent of the control subjects and 89 percent of the WAD, showing that a BPNN could be suitable for predicting motion characteristics. The statistical analysis method for purifying training samples for remote sensing classification based on BP ANN is performed. The experiments showed that it was an effective method to improve image classification. Analyses of breast cells characteristics have great importance in diagnosis, treatment and following of this disease. In this study, classification of 699 instances of breast cancer data that is available in UCI is performed through artificial neural network algorithm. Multilayer feed-forward neural network algorithm is also used for classification, application of misfire detection in gasoline engines, classification of car seat fabrics etc. however BPNN proves to be more effective than other classification algorithms.

\section{REFERENCES}

[1] Rojalina Priyadarshini; "Functional Analysis of Artificial Neural Network for Dataset Classification", Special Issue of IJCCT Vol. 1 Issue 2, 3, 4; 2010 for International Conference [ACCTA-2010], 3-5 August 2010.

[2] Guoqiang Peter Zhang "Neural Network for Classification- A Survey 2000" IEEE Transactions on systems, man and cybermetics- part c: applications and reviews, Vol 30,

[3] R.Furferi, L. Governi; "Neural Network based classification of car seat fabrics" International Journal of Mathematical Models and Methods in Applied Sciences; Issues 3, Vol. 5, 2011.

[4] E. Hosseini Aria, J. Amini, M.R.Saradjian, "Back Propagation Neural Network for Classification of IRS-1D Satellite Images" Vol.1, Issue.2, 2003

[5] Helena Grip, Fredrik Öhberg, Urban Wiklund, Ylva Sterner, J. Stefan Karlsson, and Björn Gerdle, "Classification of Neck Movement Patterns Related to Whiplash-Associated Disorders Using Neural Networks", IEEE transactions on information technology in biomedicine, Vol.7, Issue.4,2003.

[6] Yu-guo Wang, Hua-peng Li ,'Remote sensing image classification based on artificial neural network ",International Conference on Computer, Mechatronics, Control and Electronic Engineering (CMCE) , Vol.1, Issue.2, 2010.

[7] Guoqiang Peter Zhang, "Classification of Breast Cancer Data with Harmony Search and Back Propagation Based Artificial Neural Network", IEEE 22nd Signal Processing and Communications Applications Conference, 2014.

[8] Guoqiang Peter Zhang, "Neural Networks for Classification: A Survey “,IEEE transactions on systems, man, and cybernetics, Vol.30, Issue.4, 2000.

[9] Abid Ali, Olaf Magnor and Matthias Schultalbers, "Misfire Detection Using a Neural Network Based Pattern Recognition “,International Conference on Artificial Intelligence and Computational Intelligence “, Vol.2, Issue.3, 2009.

[10] R. Furferi, L. Governi and Y. Volpe, "Neural Network based classification of car seat fabrics", International journal of mathematical models and methods in applied sciences, Vol.3, Issue.5, 2011. 